\title{
Studies in East European Thought, Volume 59, Issues 1-2, 2007 Special Issue on "Dostoevskij's Significance for Philosophy and Theology"
}

\section{Robert Bird}

Published online: 9 August 2007

(C) Springer Science+Business Media B.V. 2007

During the proof procedure of this special issue not all corrections were incorporated in the final version of the papers. Please find attached a list of omitted corrections.

DOI 10.1007/s11212-007-9015-X

The Brothers Karamazov: Dostoevskij's Hosanna

Karen Stepanian

\begin{tabular}{lrll}
\hline Page No. & Line No. & Incorrect & Correct \\
\hline 92 & $12 \downarrow$ & reports that & reports \\
95 & $20 \downarrow$ & K. S.) & K. S.] \\
96 & $7 \downarrow$ & looking down & with downcast eyes \\
& $20 \downarrow$ & lower & baser \\
& $18 \uparrow$ & run & escape to \\
97 & $1 \uparrow$ & Herod: & Herod): \\
98 & $25 \uparrow$ & pass by & follow \\
100 & $12 \downarrow$ & paschal & Paschal \\
101 & $2 \downarrow$ & them of & them to be of \\
& $6 \uparrow$ & have & having \\
& $19 \downarrow$ & $96)$ Could & 96). Could \\
\hline
\end{tabular}

The online version of the original article can be found under dois: 10.1007/s11212-007-9015-x, 10.1007/ s11212-007-9018-7, 10.1007/s11212-007-9022-y, 10.1007/s11212-007-9024-9, 10.1007/s11212-0079025-8.

R. Bird $(\bowtie)$

Department of Slavic Languages \& Literatures, University of Chicago, 1130 E. 59th Street, Foster 406, Chicago, IL 60637, USA

e-mail: bird@uchicago.edu 
DOI 10.1007/s11212-007-9018-7

Unorthodox confession, orthodox conscience: aesthetic authority in the underground Sharon Lubkemann Allen

\begin{tabular}{lrll}
\hline Page No. & Line No. & Incorrect & Correct \\
\hline 82 & $5 \downarrow$ & extend & extent \\
83 & footnote 1 & Hho & Но \\
& & лўдньМ & людным \\
& & зта & эта \\
& зтот & этот \\
& & зта & эта \\
& & лу ду & люду \\
& & с! & с \\
& & с больу & с болью \\
84 & $22 \uparrow$ & margins & margins he \\
85 & $14 \uparrow$ & (re) affirmation & (re)affirmation \\
87 & $14 \uparrow$ & "irony & irony \\
& $13 \uparrow$ & flesh,” & flesh” \\
& $6 \uparrow$ & Cleopatra type & Cleopatra-type \\
89 & $4 \downarrow$ & Dostoevskji & Dostoevskij \\
& $8 \uparrow$ & Nitshche & Nitshe \\
& $7 \uparrow$ & Nietsche & Nietzsche \\
\hline
\end{tabular}

DOI 10.1007/s11212-007-9022-y

Journey to transcendence: Dostoevsky's theological polyphony in Barth's understanding of the Pauline KRISIS

Elizabeth A. Blake/Rubén Rosario

\begin{tabular}{|c|c|c|c|}
\hline Page No. & Line No. & Incorrect & Correct \\
\hline 6 & footnote $1,2 \uparrow$ & Dostoevsky & Dostoevskij \\
\hline \multirow[t]{2}{*}{7} & $16 \downarrow$ & Dostoevskij's recognition & Dostoevskij recognized \\
\hline & $16 \uparrow$ & Scleiermacher & Schleiermacher \\
\hline \multirow[t]{2}{*}{9} & $14 \uparrow$ & intends & intended \\
\hline & footnote 5 & Dostoevsky's & Dostoevskij's \\
\hline 12 & $16 \uparrow$ & {$[\ldots .$.$] not$} & {$[\ldots .$.$] not$} \\
\hline \multirow[t]{4}{*}{13} & $16 \downarrow$ & Question[....] & Question [....] \\
\hline & $10 \uparrow$ & of $[\ldots . .$.$] the$ & of $[\ldots .$.$] the$ \\
\hline & $4 \uparrow$ & Dostoevsky & Dostoevskij \\
\hline & $1 \uparrow$ & Dostoevsky & Dostoevskij \\
\hline \multirow[t]{2}{*}{14} & $1 \downarrow$ & Dostoevsky & Dostoevskij \\
\hline & $2 \downarrow$ & Dostoevsky & Dostoevskij \\
\hline 17 & $1 \uparrow$ & believe it & believe \\
\hline
\end{tabular}


DOI 10.1007/s11212-007-9024-9

Sarah Hudspith, Dostoevsky and the Idea of Russianness: A New Perspective on Unity and Brotherhood, BASEES/RoutledgeCurzon Series on Russian and East European Studies, 6. RoutledgeCurzon, London, New York, 2004, Bibliography Nel Grillaert

\begin{tabular}{lcll}
\hline Page No. & Line No. & Incorrect & Correct \\
\hline 164 & $4 \downarrow$ & is & was \\
& $10 \uparrow$ & shortly & briefly \\
165 & $6 \uparrow$ & Slavophile tinted & Slavophile-tinted \\
\hline
\end{tabular}

DOI 10.1007/s11212-007-9025-8

"Ridiculous" dream versus social contract: Dostoevskij, Rousseau, and the problem of ideal society

Olga Stuchebrukhov

\begin{tabular}{lrll}
\hline Page No. & Line No. & Incorrect & Correct \\
\hline 107 & $9 \downarrow$ & others, ${ }^{4}$ & others ${ }^{4}$ \\
110 & $23 \uparrow$ & formula & formula: \\
111 & $8 \downarrow$ & Karamzav Brothers & Brothers Karamazov \\
& $15 \downarrow$ & Chapter 2, Part I & Part I, Chapter 2 \\
115 & $18 \uparrow$ & hotenije & Khotenie \\
116 & $5 \uparrow$ & emphasis of & emphasis on \\
117 & $23 \uparrow$ & of ideal & of the ideal \\
118 & $16 \downarrow$ & a circle & the circle \\
119 & $15 \downarrow$ & a modern progressive & modern progressives \\
& $17 \downarrow$ & assertion & assertions \\
& $13 \uparrow$ & and it & and \\
120 & $9 \downarrow$ & (need, & (need \\
121 & $3 \downarrow$ & Polnoe ... tomakh & Polnoe ... tomakh \\
& $7 \uparrow$ & Ryamond & Raymond \\
\hline
\end{tabular}

